\title{
THE INFLUENCE OF NONLINEAR TERMS IN MECHANICAL SYSTEMS HAVING TWO DEGREES OF FREEDOM
}

\author{
Nguyen DuC Tinh \\ Mining Technical College, Quang ninh
}

\begin{abstract}
For many years the higher order stochastic averaging method has been widely used for investigating nonlinear systems subject to white and coloured noises to predict approximately the response of the systems. In the paper the method is further developed for two-degree-of-freedom systems subjected to white noise excitation. Application to Duffing oscillator is considered.
\end{abstract}

\section{INTRODUCTION}

It is well-known, the stochastic averaging method (SAM) is widely used in different problems of stochastic mechanics, such as vibration, stability and reliability problems (see e.g. Mitropolskii et al, 1992; Red-Horse and Spanos, 1992; Zhu and Lin, 1994; Zhu et al, 1997). However, the effect of some nonlinear terms cannot be investigated by using the classical first order SAM. In order to overcome this insufficiency the different procedures to obtain approximate solutions have been developed for the nonlinear systems with one degree of freedom under white and coloured noise excitations (see e.g. Anh, 1993; Anh and Tinh, 1995; Tinh, 1999). In the present paper this procedure is further developed for twodegree-of-freedom nonlinear systems subjected to white noise excitation. An application to Duffing system is considered and the effect of nonlinear terms can be detected in the approximate solutions of Fokker-Planck (FP) equation while it cannot be investigated by using the classical first order SAM.

\section{HIGHER SAM IN TWO-DEGREE-OF-FREEDOM SYSTEMS}

Consider the motion equations of a mechanical system with two degrees of freedom

$$
\begin{aligned}
& \ddot{x}_{1}+\omega_{1}^{2} x_{1}=\varepsilon f_{11}\left(x_{1}, x_{2}, \dot{x}_{1}, \dot{x}_{2}\right)+\varepsilon^{2} f_{12}\left(x_{1}, x_{2}, \dot{x}_{1}, \dot{x}_{2}\right)+\sqrt{\varepsilon} \sigma_{1} \dot{\xi}(t), \\
& \ddot{x}_{2}+\omega_{2}^{2} x_{2}=\varepsilon f_{21}\left(x_{1}, x_{2}, \dot{x}_{1}, \dot{x}_{2}\right)+\varepsilon^{2} f_{22}\left(x_{1}, x_{2}, \dot{x}_{1}, \dot{x}_{2}\right)+\sqrt{\varepsilon} \sigma_{2} \dot{\xi}(t),
\end{aligned}
$$

where $\omega_{1}, \omega_{2}, \sigma_{1}, \sigma_{2}$ are positive constants and $\varepsilon$ is a small positive parameter.

According to the averaging method we transform the state coordinates $x=\left(x_{1}, x_{2}\right)$ into the variables $a=\left(a_{1}, a_{2}\right)$ and $\varphi=\left(\varphi_{1}, \varphi_{1}\right)$ by the change

$$
\begin{aligned}
& x_{j}=a_{j} \cos \varphi_{j}, \\
& \dot{x}_{j}=-\omega_{j} \sin \varphi_{j}, \quad(j=1,2) .
\end{aligned}
$$


By using Ito differentiation formula [7] the system of equations (2.1) is transformed into the following system of equations

$$
\begin{aligned}
& \dot{a}_{j}=\varepsilon A_{j 1}(a, \varphi)+\varepsilon^{2} A_{j 2}(a, \varphi)-\sqrt{\varepsilon} \sigma_{j} \frac{\sin \varphi_{j}}{\omega_{j}} \dot{\xi}(t), \\
& \dot{\varphi}_{j}=\omega_{j}+\varepsilon B_{j 1}(a, \varphi)+\varepsilon^{2} B_{j 2}(a, \varphi)-\sqrt{\varepsilon} \sigma_{j} \frac{\cos \varphi_{j}}{\omega_{j} a_{j}} \dot{\xi}(t),(j=1,2)
\end{aligned}
$$

where it is denoted

$$
\begin{aligned}
& A_{j 1}(a, \varphi)=-\frac{f_{j 1}(a, \varphi)}{\omega_{j}} \sin \varphi_{j}+\frac{\sigma_{j}^{2} \cos ^{2} \varphi_{j}}{2 \omega_{j}^{2} a_{j}}, \\
& B_{j 1}(a, \varphi)=-\frac{f_{j 1}(a, \varphi)}{\omega_{j} a_{j}} \cos \varphi_{j}+\frac{\sigma_{j}^{2} \cos \varphi_{j} \sin \varphi_{j}}{\omega_{j}^{2} a_{j}^{2}}, \quad(j=1,2), \\
& A_{j 2}(a, \varphi)=-\frac{f_{j 2}(a, \varphi)}{\omega_{j}} \sin \varphi_{j}, B_{j 2}(a, \varphi)=-\frac{f_{j 2}(a, \varphi)}{\omega_{j} a_{j}} \cos \varphi_{j} .
\end{aligned}
$$

The Fokker-Planck (FP) equation for the stationary probability density function $W(a, \varphi)$ takes the form (Anh, 1995)

$$
\sum_{j=1}^{2} \omega_{j} \frac{\partial W}{\partial \varphi_{j}}=-\varepsilon\left[A_{1}, B_{1}\right] L[W]-\varepsilon^{2}\left[A_{2}, B_{2}\right] L[W],
$$

where the operators $\left[\mathrm{A}_{j}, \mathrm{~B}_{j}\right] \mathrm{L}[],. j=1,2$ are defined as follows

$$
\begin{aligned}
{\left[A_{1}, B_{1}\right] L[W]=} & \sum_{j=1}^{2}\left[\frac{\partial}{\partial a_{j}}\left(A_{j 1} W\right)+\frac{\partial}{\partial \varphi_{j}}\left(B_{j 1} W\right)\right] \\
& -\sum_{j=1}^{2} \sum_{s=1}^{2}\left\{\frac{\partial^{2}}{\partial a_{j} \partial a_{s}}\left(\frac{\sigma_{j} \sigma_{s} \sin \varphi_{j} \sin \varphi_{s}}{2 \omega_{j} \omega_{s}} W\right)\right. \\
+ & \left.\frac{\partial^{2}}{\partial a_{j} \partial \varphi_{s}}\left(\frac{\sigma_{j} \sigma_{s} \sin \varphi_{j} \cos \varphi_{s}}{2 a_{s} \omega_{j} \omega_{s}} W\right)+\frac{\partial^{2}}{\partial \varphi_{j} \partial \varphi_{s}}\left(\frac{\sigma_{j} \sigma_{s} \cos \varphi_{j} \cos \varphi_{s}}{2 a_{j} a_{s} \omega_{j} \omega_{s}} W\right)\right\} \\
& {\left[A_{2}, B_{2}\right] L[W]=\sum_{j=1}^{2}\left[\frac{\partial}{\partial a_{j}}\left(A_{j 2} W\right)+\frac{\partial}{\partial \varphi_{j}}\left(B_{j 2} W\right)\right] . }
\end{aligned}
$$

We seek the solution of (2.5) in the form

$$
W(a, \varphi)=W_{0}(a, \varphi)+\varepsilon W_{1}(a, \varphi)+\varepsilon^{2} W_{2}(a, \varphi)+\ldots
$$

Substituting (2.8) into (2.5) and comparing the coefficients of like power of $\varepsilon$ we obtain

$$
\begin{gathered}
\varepsilon^{0}: \sum_{j=1}^{2} \omega_{j} \frac{\partial W_{0}}{\partial \varphi_{j}}=0, \\
\varepsilon^{1}: \sum_{j=1}^{2} \omega_{j} \frac{\partial W_{1}}{\partial \varphi_{j}}=-\left[A_{1}, B_{1}\right] L\left[W_{0}\right],
\end{gathered}
$$




$$
\varepsilon^{2}: \sum_{j=1}^{2} \omega_{j} \frac{\partial W_{2}}{\partial \varphi_{j}}=-\left\{\left[A_{2}, B_{2}\right] L\left[W_{0}\right]+\left[A_{1}, B_{1}\right] L\left[W_{1}\right]\right\}
$$

From (2.9) we get

$$
W_{0}=W_{0}(a)
$$

The arbitrary integration function $W_{0}(a)$ must be chosen from the condition for the function $W_{1}(a, \varphi)$ to be periodic to $\varphi$.

Thus, we get from $(2.10)$

$$
<\left[A_{1}, B_{1}\right] L\left[W_{0}(a)\right]>=0,
$$

where $\langle$.$\rangle is the averaging operator with respect to \varphi$

$$
<.>=\frac{1}{(2 \pi)^{2}} \int_{0}^{2 \pi} \int_{0}^{2 \pi}(.) d \varphi_{1} d \varphi_{2}
$$

Substituting (2.6) into (2.13) yields

$$
\sum_{j=1}^{2}\left\{\frac{\partial}{\partial a_{j}}\left(<A_{j 1}>W_{0}(a)\right)-\frac{\sigma_{j}^{2}}{4 \omega_{j}^{2}} \frac{\partial^{2} W_{0}(a)}{\partial a_{j}^{2}}\right\}=0 .
$$

The second term $\mathrm{W}_{1}(\mathrm{a}, \varphi)$ in $(2.8)$ is determined from (2.10) using Fourier expansion

$$
\left[A_{1}, B_{1}\right] L\left[W_{0}(a)\right]=W_{0}(a) \sum_{l_{1}} \sum_{l_{2}} C_{l_{1} l_{2}}(a) \exp \left[i\left(l_{1} \varphi_{1}+l_{2} \varphi_{2}\right)\right]
$$

where

$$
C_{l_{1} l_{2}}(a)=\frac{1}{(2 \pi)^{2} W_{0}(a)} \int_{0}^{2 \pi} \int_{0}^{2 \pi}\left[A_{1}, B_{1}\right] L\left[W_{0}(a)\right] \exp \left[-i\left(l_{1} \varphi_{1}+l_{2} \varphi_{2}\right)\right] d \varphi_{1} d \varphi_{2} .
$$

Substituting (2.16) into (2.10) we get

$$
W_{1}(a, \varphi)=W_{0}(a)\left\{W_{10}(a)+\sum_{l_{1}} \sum_{l_{2}} \frac{C_{l_{1} l_{2}}}{l_{1} \omega_{1}+l_{2} \omega_{2}} \exp \left[i\left(l_{1} \varphi_{1}+l_{2} \varphi_{2}\right)\right\},\right.
$$

where $l_{1}, l_{2}$ are integers and

$$
l_{1} \omega_{1}+l_{2} \omega_{2} \neq 0
$$

The arbitrary integration function $W_{10}(a)$ must be chosen from the condition for the function $W_{2}(a, \varphi)$ to be periodic to $\varphi$. Similarly, we can find the third term $W_{2}(a, \varphi)$ in (2.11). 


\section{APPLICATION}

\subsection{SAM of coefficients in FP equation}

Now we apply the proposed procedure to Duffing system whose motion equations take the form

$$
\begin{aligned}
& m_{1} \ddot{x}_{1}+c_{1} x_{1}=-\varepsilon\left[2 h_{1} \dot{x}_{1}+\beta_{1} x_{1}^{3}\right]-\varepsilon^{2}\left[c_{12}\left(x_{1}-x_{2}\right)+2 h_{12}\left(\dot{x}_{1}-\dot{x}_{2}\right)\right]+\sqrt{\varepsilon} \delta_{1} \dot{\xi}(t), \\
& m_{2} \ddot{x}_{2}+c_{2} x_{2}=-\varepsilon\left[2 h_{2} \dot{x}_{2}+\beta_{2} x_{2}^{3}\right]+\varepsilon^{2}\left[c_{12}\left(x_{1}-x_{2}\right)+2 h_{12}\left(\dot{x}_{1}-\dot{x}_{2}\right)\right]+\sqrt{\varepsilon} \delta_{2} \dot{\xi}(t) .
\end{aligned}
$$

We represent the physical model of this system in Fig. 1 . Where $m_{1}, m_{2}$ are masses, $c_{1}$, $c_{2}, c_{12}$ are spring constants, $h_{1}, h_{2}, h_{12}$ are damping coefficients, $\beta_{1}, \beta_{2}, \delta_{1}, \delta_{2}$ are positive constants and

$$
R_{1}=-\varepsilon \beta_{1} x_{1}^{3}+\sqrt{\varepsilon} \delta_{1} \dot{\xi}(t), \quad R_{2}=-\varepsilon \beta_{2} x_{2}^{3}+\sqrt{\varepsilon} \delta_{2} \dot{\xi}(t) .
$$

The system of equations (3.1) can be written in the form

$$
\begin{aligned}
& \ddot{x}_{1}+\omega_{1}^{2} x_{1}=-\varepsilon\left[2 k_{1} \dot{x}_{1}+\gamma_{1} x_{1}^{3}\right]-\varepsilon^{2}\left[q_{1}\left(x_{1}-x_{2}\right)+2 k_{11}\left(\dot{x}_{1}-\dot{x}_{2}\right)\right]+\sqrt{\varepsilon} \sigma_{1} \dot{\xi}(t), \\
& \ddot{x}_{2}+\omega_{2}^{2} x_{2}=-\varepsilon\left[k_{2} \dot{x}_{2}+\gamma_{2} x_{2}^{3}\right]+\varepsilon^{2}\left[q_{2}\left(x_{1}-x_{2}\right)+2 k_{12}\left(\dot{x}_{1}-\dot{x}_{2}\right)\right]+\sqrt{\varepsilon} \sigma_{2} \dot{\xi}(t),
\end{aligned}
$$

where

$$
\begin{aligned}
& \omega_{1}^{2}=\frac{c_{1}}{m_{1}}, \omega_{2}^{2}=\frac{c_{2}}{m_{2}}, k_{1}=\frac{h_{1}}{m_{1}}, k_{2}=\frac{h_{2}}{m_{2}}, \gamma_{1}=\frac{\beta_{1}}{m_{1}}, \gamma_{2}=\frac{\beta_{2}}{m_{2}}, \\
& q_{1}=\frac{c_{12}}{m_{1}}, q_{2}=\frac{c_{12}}{m_{2}}, k_{11}=\frac{h_{12}}{m_{1}}, k_{12}=\frac{h_{12}}{m_{2}}, \sigma_{1}=\frac{\delta_{1}}{m_{1}}, \sigma_{2}=\frac{\delta_{2}}{m_{2}} \text {. } \\
& { }_{R_{2}}
\end{aligned}
$$

Fig. 1. Physical model of a mechanical system with two degrees of freedom

In this case we have

$$
\begin{aligned}
& f_{11}=-2 k_{1} \dot{x}_{1}-\gamma x_{1}^{3} ; \quad f_{12}=-q_{1}\left(x_{1}-x_{2}\right)-2 k_{11}\left(\dot{x}_{1}-\dot{x}_{2}\right) ; \\
& f_{21}=-2 k_{2} \dot{x}_{2}-\gamma x_{2}^{3} ; \quad f_{22}=q_{2}\left(x_{1}-x_{2}\right)+2 k_{12}\left(\dot{x}_{1}-\dot{x}_{2}\right) .
\end{aligned}
$$

From (2.4), using (2.2) and (3.5), after calculations we obtain

$$
\begin{aligned}
& A_{11}=-2 k_{1} a_{1} \sin ^{2} \varphi_{1}+\frac{\gamma_{1}}{\omega_{1}} a_{1}^{3} \sin \varphi_{1} \cos ^{3} \varphi_{1}+\frac{\sigma_{1}^{2}}{2 \omega_{1}^{2} a_{1}} \cos ^{2} \varphi_{1}, \\
& A_{21}=-2 k_{2} a_{2} \sin ^{2} \varphi_{2}+\frac{\gamma_{2}}{\omega_{2}} a_{2}^{3} \sin \varphi_{2} \cos ^{3} \varphi_{2}+\frac{\sigma_{2}^{2}}{2 \omega_{2}^{2} a_{2}} \cos ^{2} \varphi_{2},
\end{aligned}
$$




$$
\begin{aligned}
& B_{11}=-k_{1} \sin 2 \varphi_{1}+\frac{\gamma_{1} a_{1}^{2}}{\omega_{1}} \cos ^{4} \varphi_{1}-\frac{\sigma_{1}^{2}}{2 \omega_{1}^{2} a_{1}^{2}} \sin 2 \varphi_{1} \\
& B_{21}=-k_{2} \sin 2 \varphi_{2}+\frac{\gamma_{2} a_{2}^{2}}{\omega_{2}} \cos ^{4} \varphi_{2}-\frac{\sigma_{2}^{2}}{2 \omega_{2}^{2} a_{2}^{2}} \sin 2 \varphi_{2}
\end{aligned}
$$

$$
\begin{aligned}
& A_{12}=\frac{q_{1}}{\omega_{1}}\left(a_{1} \cos \varphi_{1}-a_{2} \cos \varphi_{2}\right) \sin \varphi_{1}+\frac{2 k_{11}}{\omega_{1}}\left(-\omega_{1} a_{1} \sin ^{2} \varphi_{1}+\omega_{2} a_{2} \sin \varphi_{1} \sin \varphi_{2}\right), \\
& A_{22}=\frac{q_{2}}{\omega_{2}}\left(-a_{1} \cos \varphi_{1}+a_{2} \cos \varphi_{2}\right) \sin \varphi_{2}+\frac{2 k_{12}}{\omega_{2}}\left(-\omega_{2} a_{2} \sin ^{2} \varphi_{2}+\omega_{1} a_{1} \sin \varphi_{1} \sin \varphi_{2}\right), \\
& B_{12}=\left[\frac{q_{1}}{\omega_{1} a_{1}}\left(a_{1} \cos \varphi_{1}-a_{2} \cos \varphi_{2}\right)+\frac{2 k_{11}}{\omega_{1} a_{1}}\left(-\omega_{1} a_{1} \sin \varphi_{1}+\omega_{2} a_{2} \sin \varphi_{2}\right)\right] \cos \varphi_{1}, \\
& B_{22}=\frac{q_{2}}{\omega_{2} a_{2}}\left[\left(-a_{1} \cos \varphi_{1}+a_{2} \cos \varphi_{2}\right)+\frac{2 k_{12}}{\omega_{2} a_{2}}\left(-\omega_{2} a_{2} \sin \varphi_{2}+\omega_{1} a_{1} \sin \varphi_{1}\right)\right] \sin \varphi_{2},
\end{aligned}
$$

From (3.6) and (3.7), using (2.14) we get

$$
\begin{gathered}
<A_{11}>=-k_{1} a_{1}+\frac{\sigma_{1}^{2}}{4 \omega_{1}^{2} a_{1}} ; \quad<A_{21}>=-k_{2} a_{2}+\frac{\sigma_{2}^{2}}{4 \omega_{2}^{2} a_{2}} \\
<A_{12}>=-k_{11} a_{1} ; \quad<A_{22}>=-k_{12} a_{2}
\end{gathered}
$$

In this case, from (2.5) the averaged FP equation takes the form

$$
\left[<A_{1}, B_{1}>\right] L[W(a)]+\varepsilon\left[<A_{2}, B_{2}>\right] L[W(a)]=0,
$$

where it is denoted

$$
\begin{gathered}
{\left[<A_{1}, B_{1}>\right] L[W(a)]=\sum_{j=1}^{2}\left[\frac{\partial}{\partial a_{j}}\left(<A_{j 1}>W(a)\right)+\frac{\partial}{\partial \varphi_{j}}\left(<B_{j 1}>W(a)\right)\right]} \\
\quad-\sum_{j=1}^{2} \sum_{s=1}^{2}\left\{\frac{\partial^{2}}{\partial a_{j} \partial a_{s}}\left(\left\langle\frac{\sigma_{j} \sigma_{s} \sin \varphi_{j} \sin \varphi_{s}}{2 \omega_{j} \omega_{s}}\right\rangle W(a)\right)\right. \\
\left.+\frac{\partial^{2}}{\partial a_{j} \partial \varphi_{s}}\left(\left\langle\frac{\sigma_{j} \sigma_{s} \sin \varphi_{j} \cos \varphi_{s}}{2 a_{s} \omega_{j} \omega_{s}}\right\rangle W(a)\right)+\frac{\partial^{2}}{\partial \varphi_{j} \partial \varphi_{s}}\left(\left\langle\frac{\sigma_{j} \sigma_{s} \cos \varphi_{j} \cos \varphi_{s}}{2 a_{j} a_{s} \omega_{j} \omega_{s}}\right\rangle W(a)\right)\right\} \\
{\left[<A_{2}, B_{2}>\right] L[W(a)]=\sum_{j=1}^{2}\left[\frac{\partial}{\partial a_{j}}\left(<A_{j 2}>W(a)\right)+\frac{\partial}{\partial \varphi_{j}}\left(<B_{j 2}>W(a)\right)\right]}
\end{gathered}
$$

From (3.9), noting (3.8) and (3.10) we have the FP equation for the probability density function $W(a)$ in the form

$$
\sum_{j=1}^{2}\left\{\frac{\partial}{\partial a_{j}}\left[\left(-k_{j} a_{j}+\frac{\sigma_{j}^{2}}{4 \omega_{j}^{2} a_{j}}-\varepsilon k_{1 j} a_{j}\right) W(a)\right]-\frac{\sigma_{j}^{2}}{4 \omega_{j}^{2}} \frac{\partial^{2} W(a)}{\partial a_{j}^{2}}\right\}=0 .
$$


This equation gives the solution

$$
\begin{aligned}
& W(a)=C a_{1} a_{2} \exp \left\{-\frac{2 k_{1} \omega_{1}^{2}}{\sigma_{1}^{2}} a_{1}^{2}-\frac{2 k_{2} \omega_{2}^{2}}{\sigma_{2}^{2}} a_{2}^{2}-\varepsilon\left(\frac{2 k_{11} \omega_{1}^{2}}{\sigma_{1}^{2}} a_{1}^{2}+\frac{2 k_{12} \omega_{2}^{2}}{\sigma_{2}^{2}} a_{2}^{2}\right)\right\}, \\
& (C=\text { const }) .
\end{aligned}
$$

The condition

$$
\int_{0}^{2 \pi} \int_{0}^{2 \pi} \int_{0}^{\infty} \int_{0}^{\infty} W(a) d a_{1} d a_{2} d \varphi_{1} d \varphi_{2}=1
$$

gives

$$
C=\frac{\left(p_{1}+\varepsilon \alpha_{1}\right)\left(p_{2}+\varepsilon \alpha_{2}\right)}{\pi^{2}}
$$

where

$$
p_{1}=\frac{2 k_{1} \omega_{1}^{2}}{\sigma_{1}^{2}}, p_{2}=\frac{2 k_{2} \omega_{2}^{2}}{\sigma_{2}^{2}}, \alpha_{1}=\frac{2 k_{11} \omega_{1}^{2}}{\sigma_{1}^{2}}, \alpha_{2}=\frac{2 k_{12} \omega_{2}^{2}}{\sigma_{2}^{2}} .
$$

It can be seen from (3.12) that the effect of the nonlinear terms $\varepsilon \beta_{1} x_{1}^{3}$ and $\varepsilon \beta_{2} x_{2}^{3}$ is lost during the conventional averaging procedure and we can not show their effect in the approximation (3.12) of the density probability function $W(a, \varphi)$. So, in order to overcome this insufficiency we need to determine the higher approximate solutions to the FP equation by using the higher SAM.

\subsection{Higher approximate solutions to FP equation}

We determine the second approximate solution of FP equation (2.5) for the Duffing system (3.1).

Substituting (3.8) into (2.15) yields

$$
W_{0}(a)=C a_{1} a_{2} \exp \left\{-\frac{2 k_{1} \omega_{1}^{2}}{\sigma_{1}^{2}} a_{1}^{2}-\frac{2 k_{2} \omega_{2}^{2}}{\sigma_{2}^{2}} a_{2}^{2}\right\}, \quad(C=\text { const }) .
$$

Substituting (3.15) into (2.10) we get

$$
\sum_{j=1}^{2} \omega_{j} \frac{\partial W_{1}}{\partial \varphi_{j}}=-\left[A_{1}, B_{1}\right] L\left[W_{0}(a)\right]
$$

Using (2.6), (3.6) and expanding the right-hand side of (3.16) into the double Fourier series of $\varphi_{1}, \varphi_{2}$, after calculations we have the equation for $W_{11}(a, \varphi)$

$$
\begin{gathered}
\omega_{1} \frac{\partial W_{11}}{\partial \varphi_{1}}+\omega_{2} \frac{\partial W_{11}}{\partial \varphi_{2}}=2 k_{1} \cos \varphi_{1}+2 k_{2} \cos \varphi_{2}+\frac{k_{1} \omega_{1} \gamma_{1}}{2 \sigma_{1}^{2}} a_{1}^{4}\left(2 \sin \varphi_{1}+\sin 4 \varphi_{1}\right) \\
+\frac{k_{2} \omega_{2} \gamma_{2}}{2 \sigma_{2}^{2}} a_{2}^{4}\left(2 \sin \varphi_{2}+\sin 4 \varphi_{2}\right)+\frac{s(a)}{2} \cos \left(\varphi_{1}-\varphi_{2}\right)+\frac{s(a)}{2} \cos \left(\varphi_{1}+\varphi_{2}\right) .
\end{gathered}
$$


Using the principle of superposition we get the solution of equation (3.17)

$$
\begin{aligned}
W_{11}(a, \varphi)= & \frac{k_{1}}{\omega_{1}} \sin 2 \varphi_{1}+\frac{k_{2}}{\omega_{2}} \sin 2 \varphi_{2}-\frac{k_{1} \gamma_{1}}{8 \sigma_{1}^{2}} a_{1}^{4}\left(4 \cos 2 \varphi_{1}+\cos 4 \varphi_{1}\right) \\
& -\frac{k_{2} \gamma_{2}}{8 \sigma_{2}^{2}} a_{2}^{4}\left(4 \cos 2 \varphi_{2}+\cos 4 \varphi_{2}\right)+\frac{\omega_{1}}{\omega_{2}^{2}-\omega_{1}^{2}} s(a) \cos \varphi_{1} \sin \varphi_{2} \\
& -\frac{\omega_{2}}{\omega_{2}^{2}-\omega_{1}^{2}} s(a) \sin \varphi_{1} \cos \varphi_{2},
\end{aligned}
$$

where

$$
s(a)=\frac{\sigma_{1} \sigma_{2}}{\omega_{1} \omega_{2}} \frac{1}{a_{1} a_{2}}-\frac{2 k_{1} \omega_{1} \sigma_{2}}{\omega_{2} \sigma_{1}} \frac{a_{1}}{a_{2}}-\frac{2 k_{2} \omega_{2} \sigma_{1}}{\omega_{1} \sigma_{2}} \frac{a_{2}}{a_{1}}+\frac{16 k_{1} k_{2} \omega_{1} \omega_{2}}{\sigma_{1} \sigma_{2}} a_{1} a_{2} .
$$

Substituting (3.15) and (3.18) into (2.11) we have the equation for the arbitrary function $\mathrm{W}_{10}(\mathrm{a})$ in the form

$$
\begin{aligned}
& \left.\sum_{j=1}^{2} \frac{\partial}{\partial a_{j}}\left(<A_{j 1}>W_{0} W_{10}\right)-\sum_{j=1}^{2} \frac{\partial^{2}}{\partial a_{j}^{2}}\left(\frac{\sigma_{j}^{2}}{4 \omega_{j}^{2}} W_{0} W_{10}\right)=\sum_{j=1}^{2} \frac{\partial^{2}}{\partial a_{j}^{2}}\left(\frac{\sigma_{j}^{2}}{2 \omega_{j}^{2}}<W_{0} W_{11} \sin ^{2} \varphi_{j}\right\rangle\right) \\
& \left.-\sum_{j=1}^{2} \frac{\partial}{\partial a_{j}}\left(<A_{j 1} W_{0} W_{11}\right\rangle\right)-\sum_{j=1}^{2} \frac{\partial}{\partial a_{j}}\left(<A_{j 2}>W_{0}\right) .
\end{aligned}
$$

Substituting $A_{j 1}$ in (3.6), $A_{j 2}$ in $(3.7),(j=1,2)$ and $W_{11}(a, \varphi)$ in (3.18) into (3.20), after calculations we have the equation (3.20) in the form

$$
\begin{gathered}
\sum_{j=1}^{2} \frac{\partial}{\partial a_{j}}\left[\frac{\sigma_{j}^{2}}{4 \omega_{j}^{2}} \frac{\partial W_{10}}{\partial a_{j}} W_{0}(a)\right]= \\
-\sum_{j=1}^{2}\left\{\frac{\partial^{2}}{\partial a_{j}^{2}}\left(\frac{k_{j} \gamma_{j}}{16 \omega_{j}^{2}} a_{j}^{4} W_{0}(a)+\frac{\partial}{\partial a_{j}}\left[\left(\frac{k_{j}^{2} \gamma_{j}}{4 \sigma_{j}^{2}}+\frac{k_{j} \gamma_{j}}{16 \omega_{j}^{2}}\right) W_{0}(a)\right]+\frac{\partial}{\partial a_{j}}\left[k_{1 j} a_{j} W_{0}(a)\right]\right\} .\right.
\end{gathered}
$$

The solution $W_{10}(a)$ of equation (3.21) can be found in the form

$$
W_{10}(a)=W_{01}\left(a_{1}\right)+W_{02}\left(a_{2}\right),
$$

Substituting (3.22) into (3.21), after calculations we get

$$
W_{10}(a)=-\alpha_{1} a_{1}^{2}-\alpha_{2} a_{2}^{2}-\alpha_{11} a_{1}^{4}-\alpha_{22} a_{2}^{4}
$$

where $\alpha_{1}, \alpha_{2}$ are defined in (3.14) and

$$
\alpha_{11}=\frac{3 k_{1} \gamma_{1}}{8 \sigma_{1}^{2}}, \alpha_{22}=\frac{3 k_{2} \gamma_{2}}{8 \sigma_{2}^{2}}
$$

Hence, using the second approximate solution to the FP equation (2.5) for the Duffing system (3.1) takes the form

$$
W(a, \varphi)=W_{0}(a)\left\{1+\varepsilon\left[W_{10}(a)+W_{11}(a, \varphi)\right]\right\},
$$

where $W_{0}(a), W_{11}(a, \varphi)$ and $W_{10}(a)$ are defined in (3.15), (3.18) and (3.23), respectively. It is seen from (3.18) and (3.23) that the effect of the nonlinear terms $\varepsilon \beta_{1} x_{1}^{3}$ and $\varepsilon \beta_{2} x_{2}^{3}$ is shown in the formula (3.25).

From the normalization condition 
we get

$$
\int_{0}^{2 \pi} \int_{0}^{2 \pi} \int_{0}^{\infty} \int_{0}^{\infty} W(a, \varphi) d a_{1} d a_{2} d \varphi_{1} d \varphi_{2}=1
$$

$$
C=\frac{p_{1}^{3} p_{2}^{3}}{\pi^{2}\left[p_{1}^{2} p_{2}^{2}-\varepsilon\left(\alpha_{1} p_{1} p_{2}^{2}+\alpha_{2} p_{1}^{2} p_{2}+\alpha_{11} p_{2}^{2}+\alpha_{22} p_{1}^{2}\right)\right]} .
$$

Now we find the approximate mean squares $E\left[x_{1}^{2}\right]$ and $E\left(x_{2}^{2}\right)$ for the cases of the classical and higher SAM.

In the case of the classical SAM we have

$$
E_{c l}\left[x_{j}^{2}\right]=\int_{0}^{2 \pi} \int_{0}^{2 \pi} \int_{0}^{\infty} \int_{0}^{\infty} x_{j}^{2} W(a) d a_{1} d a_{2} d \varphi_{1} d \varphi_{2}
$$

Substituting $x_{j},(\mathrm{j}=1,2)$ in $(2.2), W(a)$ in (3.12) and $C$ in (3.13) into (3.27), after calculations we have

$$
E_{c l}\left[x_{1}^{2}\right]=\frac{1}{2\left(p_{1}+\varepsilon \alpha_{1}\right)}=\frac{1}{2 p_{1}}-\varepsilon \frac{\alpha_{1}}{p_{1}^{2}}+\varepsilon^{2} \ldots
$$

Similarly, we have

$$
E_{c l}\left[x_{2}^{2}\right]=\frac{1}{2\left(p_{2}+\varepsilon \alpha_{2}\right)}=\frac{1}{2 p_{2}}-\varepsilon \frac{\alpha_{2}}{p_{2}^{2}}+\varepsilon^{2} \ldots
$$

where $p_{1}, \mathrm{p}_{2}, \alpha_{1}$ and $\alpha_{2}$ are defined in (3.14).

In the case of the higher SAM we have

$$
E\left[x_{j}^{2}\right]=\int_{0}^{2 \pi} \int_{0}^{2 \pi} \int_{0}^{\infty} \int_{0}^{\infty} x_{j}^{2} W(a, \varphi) d a_{1} d a_{2} d \varphi_{1} d \varphi_{2}, \quad(j=1,2) .
$$

Substituting $x_{j},(j=1,2)$ in $(2.2), W(a, \varphi)$ in (3.25) and $C$ in (3.26) into (3.30), noting (3.24), after calculations we have

$$
E\left[x_{1}^{2}\right]=\frac{p_{1}^{2} p_{2}^{2}-\varepsilon\left(2 \alpha_{1} p_{1} p_{2}^{2}+\alpha_{2} p_{1}^{2} p_{2}+10 \alpha_{11} p_{2}^{2}+2 \alpha_{22} p_{1}^{2}\right)}{2 p_{1}\left[p_{1}^{2} p_{2}^{2}-\varepsilon\left(\alpha_{1} p_{1} p_{2}^{2}+\alpha_{2} p_{1}^{2} p_{2}+\alpha_{11} p_{2}^{2}+\alpha_{22} p_{1}^{2}\right)\right]} .
$$

Expanding the right-hand side of (3.31) into the power series of $\varepsilon$ we get

$$
E\left[x_{1}^{2}\right]=\frac{1}{2 p_{1}}-\varepsilon\left(\frac{\alpha_{1}}{2 p_{1}^{2}}+\frac{9 \alpha_{11}}{2 p_{1}^{3}}+\frac{\alpha_{22}}{2 p_{1} p_{2}^{2}}\right)+\varepsilon^{2} \ldots
$$

Similarly, we have

$$
E\left[x_{2}^{2}\right]=\frac{1}{2 p_{2}}-\varepsilon\left(\frac{\alpha_{2}}{2 p_{2}^{2}}+\frac{9 \alpha_{22}}{2 p_{2}^{3}}+\frac{\alpha_{11}}{2 p_{1}^{2} p_{2}}\right)+\varepsilon^{2} \ldots
$$

Hence, the second approximate solution to the FP equation, the effect of the nonlinear terms $\varepsilon \beta_{1} x_{1}^{3}$ and $\varepsilon \beta_{2} x_{2}^{3}$ is obtained in the formulae (3.32) and (3.33). It is seen from (3.32) and (3.33) that the nonlinear terms $\varepsilon \beta_{1} x_{1}^{3}$ and $\varepsilon \beta_{2} x_{2}^{3}$ reduce the mean squares $E\left[x_{1}^{2}\right]$ and $E\left[x_{2}^{2}\right]$.

In the case $\beta_{1}=\beta_{2}=0$ (linear system) we have

$$
E\left[x_{1}^{2}\right]=\frac{1}{2 p_{1}}-\varepsilon \frac{\alpha_{1}}{2 p_{1}^{2}}+\varepsilon^{2} \ldots, E\left[x_{2}^{2}\right]=\frac{1}{2 \alpha_{2}}-\varepsilon \frac{\alpha_{2}}{2 p_{2}^{2}}+\varepsilon^{2} \ldots
$$


which equal to the mean squares in (3.28), (3.29) obtained by the classical SAM.

We consider the case where $m_{1}=m_{2}=c_{1}=c_{2}=\delta_{1}=\delta_{2}=1, \beta_{1}=\beta_{2}=\beta$, $\mathrm{h}_{1}=\mathrm{h}_{2}=h_{12}=c_{12}=0.5$. The mean square responses corresponding to some values of the coefficients $\beta$ are given in Table 1. It is seen that the mean square responses decrease when increasing the coefficient $\beta$.

Table 1. Mean square responses to Duffing system

(Effect of non-linear coefficient $\beta$ )

\begin{tabular}{|c|c|c|}
\hline$N$ & $\beta$ & $E\left[x_{1}^{2}\right]=E\left[x_{2}^{2}\right]$ \\
\hline 1 & 0 & $0.5-0.5 \varepsilon+\varepsilon^{2} \ldots$ \\
2 & 0.2 & $0.5-0.6875 \varepsilon+\varepsilon^{2} \ldots$ \\
3 & 0.5 & $0.5-0.9687 \varepsilon+\varepsilon^{2} \ldots$ \\
4 & 1.0 & $0.5-1.4375 \varepsilon+\varepsilon^{2} \ldots$ \\
5 & 2.0 & $0.5-2.375 \varepsilon+\varepsilon^{2} \ldots$ \\
\hline
\end{tabular}

\section{CONCLUSION}

For many years the higher order stochastic averaging method has been widely used for investigating single-degree-of-freedom nonlinear systems subject to white and coloured random noises. In this paper, the method is applied to the nonlinear vibration systems having two degrees of freedom under white noise excitations. The application to the Duffing system is considered and shows the effect of the non-linear terms to the mean square responses of the system.

Acknowledgement. Support from the Council for natural sciences of Vietnam is gratefully acknowledged.

\section{REFERENCES}

1. N. D. Anh, Higher order approximate splutions in stochastic averaging method, In Proc. of NCSR of VN 5 (1993) 19-26.

2. N. D. Anh, Higher order averaging method of coefficients in Fokker- Planck equation, In special volume: Advances in Nonlinear Structural Dynamics of SADHANA, Indian Acad. of Sc. (1995) 373-388.

3. N. D. Anh and N. D. Tinh, Higher order averaging solutions for Van-Der-Pol oscillatior, In Proc. Int. Conf. on Nonlinear Stochastic Dynamics, Hanoi,Vietnam (1995) 27-38.

4. N. D. Anh and N. D. Tinh, The influence of second order narrow-band colored noises on non-linear random vibrations, In Vietnam Journal of Mechanics 21 (1999) 65-74.

5. S. T. Ariaratnam, D. S. F. Tam, Random vibration and stability of a linear parametrically excited oscillator, $Z$. Andrew. Math. Mech. 59 (1979) 79-84.

6. R. A. Ibrahim, Parametric Random Vibration, (Hertfordsire New York: Research Studies Press/John Wiley and Sons) 1985.

7. A. Mitropolskii Iu, N. V. Dao, N. D. Anh, Nonlinear Oscillations in Systems of Arbitrary Order, Naukova-Dumka, Kiev, 1992 (In Russian).

8. J. R. Red-Horse, Spanos P. T. D., A generalization to stochastic averaging in random vibration, Int. J. Nonlinear Mech. 27 (1992) 85-101-134.

9. J. B. Roberts, and P. T. D. Spanos, Stochastic averaging: An approximate method of solving random problem, Int. J. Nonlinear Mech. 21 (1986) 111-134. 
10. N. D. Tinh, Higher order stochastic averaging method in Nonlinear vibration Systems, Doctorate Diploma, Hanoi, 1999 (In Vietnamese).

11. N. D. Tinh, Higher order stochastic averaging method for mechanical systems having two degrees of freedom, In Vietnam Journal of Mechanics 26 (2) (2004) 103-110.

12. W. Q. Zhu, Stochastic averaging method in random vibrations, Appl. Mech. Rev. 41 (1988) 189-199.

13. W. Q. Zhu, M. Q. Yu, Y. K. Lin, On improved stochastic averaging procedure, Probab. Eng. Mech. 9 (1994) 203-212.

14. W. Q. Zhu et al., Stochastic averaging of quasi-integrable Hamintonian systems, $J$. Applied Mech. 64 (1997) 975-984.

Received March 20, 2006

Revised May 18, 2006

\section{ÅNH HƯ ̛̛̉NG CƯA SỐ HẠNG PHI TUYẾN TRONG CÁC HỆ Cơ HỌC HAI BẬC TƯ DO}

Phương pháp trung bình ngẫu nhiên bậc nhất được áp dụng rộng rãi đối với các hệ dao động phi tuyển chịu kích động ngẫu nhiên dạng ồn trẳng và ồn màu. Tuy nhiên, hiệu ứng của nhiều số hạng phi tuyến bị biến mất do kết quả của phép lấy trung bình. Để khắc phục nhược điểm trên phương pháp trung bình ngấu nhiên bậc cao được phát triển. Trong bài báo, phương pháp được trình bày đối với hệ phi tuyến yếu hai bậc tự do chịu kích đệng ngẫu nhiên dạng ồn trắng. Sau đó phương pháp được áp dụng để xác định nghiệm xấp xỉ bậc hai của phương trình Fokker-Planck đối với hệ dạng Duffing. 